\title{
Numerical study on the spatial extent of interaction region surrounding comet nucleus-Ion pick-up process
}

\author{
Yuto Katoh ${ }^{1 *}$, Hiroshi Oya ${ }^{2}$, Masahide Iizima ${ }^{1}$, and Takayuki Ono ${ }^{1}$ \\ ${ }^{1}$ Department of Geophysics, Graduate School of Science, Tohoku University, 980-8578, Japan \\ ${ }^{2}$ Fukui University of Technology, 910-4272, Japan
}

(Received July 15, 2003; Revised October 26, 2003; Accepted October 28, 2003)

\begin{abstract}
The observations made by Sakigake revealed the asymmetric distribution of the interaction region of Halley's comet, and the center of the interaction region was shifted toward the post-encounter side on the spacecraft trajectory. Oya (1993) proposed the model that this asymmetric distribution is explained by the field aligned motion of picked up ions. Based on this model, we examined spatial and time scale of field aligned motion of cometary ions in the ion pick-up process by using numerical experiments and the shift of the interaction region is estimated. In the simulation results, it was shown that the braking process of the field aligned motion of picked up ions are understood by the wave particle interaction through the excitation of the MHD wave and pitch angle scattering caused by the turbulent magnetic field, and spatial scale of field aligned motion of cometary ion was estimated to $1.5 \times 10^{6} \mathrm{~km}$. This result suggests that observed asymmetrical distribution of the interaction region of the comet is formed by the motion of picked up ions toward the field aligned direction until their merging into the solar wind, as it was proposed by Oya (1993).
\end{abstract}

Key words: Comet-solar wind interaction, ion pick-up, ion beam instability, hybrid simulation.

\section{Introduction}

Since the achievement of the international collaboration of Comet Halley observation (IHW in 1986-1987), there have been many remarkable studies on the interaction of the cometary plasma with the solar wind. Based on results of the plasma wave experiments made by Sakigake for Comet Halley, Oya (1993) states that the plasma turbulence region distributes with a very clear asymmetrical feature with respect to the nucleus of Halley's Comet; plasma turbulence was first observed at the distance of $10^{7} \mathrm{~km}$ from the nucleus in the pre-encounter side, and extended to the distance of $2.2 \times 10^{7} \mathrm{~km}$ in the post-encounter side. Since the observational results showed that the center of interaction region was shifted $5 \times 10^{6} \mathrm{~km}$ toward the post-encounter side, Oya (1993) proposed a model that this asymmetric distribution is caused by an obliqueness of interplanetary magnetic field with respect to the direction of solar wind plasma flow, as shown in Fig. 1. In this model, cometary ions move along the magnetic field direction and this field aligned motion makes asymmetrical distribution in a plane which is parallel to the magnetic field line till merging into solar wind. This asymmetric picture was also observed by ion detector onboard Giotto (McKenna-Lawlor et al., 1986).

In the present paper we study on the wave particle interaction process carried out in the ion pick-up process examining nonlinear evolution of electromagnetic waves in the Comet-

*Present address: Radio Science Center for Space and Atmosphere, Kyoto University, 611-0011, Japan.

Copy right(c) The Society of Geomagnetism and Earth, Planetary and Space Sciences (SGEPSS); The Seismological Society of Japan; The Volcanological Society of Japan; The Geodetic Society of Japan; The Japanese Society for Planetary Sciences.
Coma region extended up to a scale size of $10^{7} \mathrm{~km}$. Spatial scale size of asymmetry of Comet-Coma region is determined by the scale of field aligned motion of picked up ions. Trapped cometary ions are finally merged into the solar wind plasma flow due to the nonlinear wave particle interaction process. To explain the asymmetric distribution of cometary ion, it is necessary to understand the quantitative character of the ion pick-up process inside the Comet-Coma region. Our purpose in this paper is to investigate the braking process of the field aligned motion of picked up ions which directly determines the spatial scale of the asymmetry of Comet-Coma region.

There have been many theoretical studies and numerical experiments of the wave particle interaction caused by the ion beam instability (e.g. Gary et al., 1986; Omidi and Winske, 1987) concerning the ion pick-up process near the cometary coma region. They achieved the basic understanding of ion pick up process; namely, the large amplitude MHD wave is excited by ion beam instability where cometary ions act as energy source of waves. Large amplified MHD wave induces the daughter waves through its decay process (Terasawa et al., 1986; Kojima et al., 1989) and turbulent state is generated in the upstream of the comet (Krauklis and Johnstone, 1998). Near the cometary shock, in those scenario, picked up ions are thermalized through the interaction with the turbulent fluctuations and form shell distribution in the velocity space (Wu et al., 1986; Karimabadi et al., 1994).

Generation process of MHD waves and particle motions in the vicinity of the cometary shock region has been well investigated, however, it remains to be studied about details of field aligned motion and merging process of cometary ions into solar wind plasma. This problem requires a numerical 

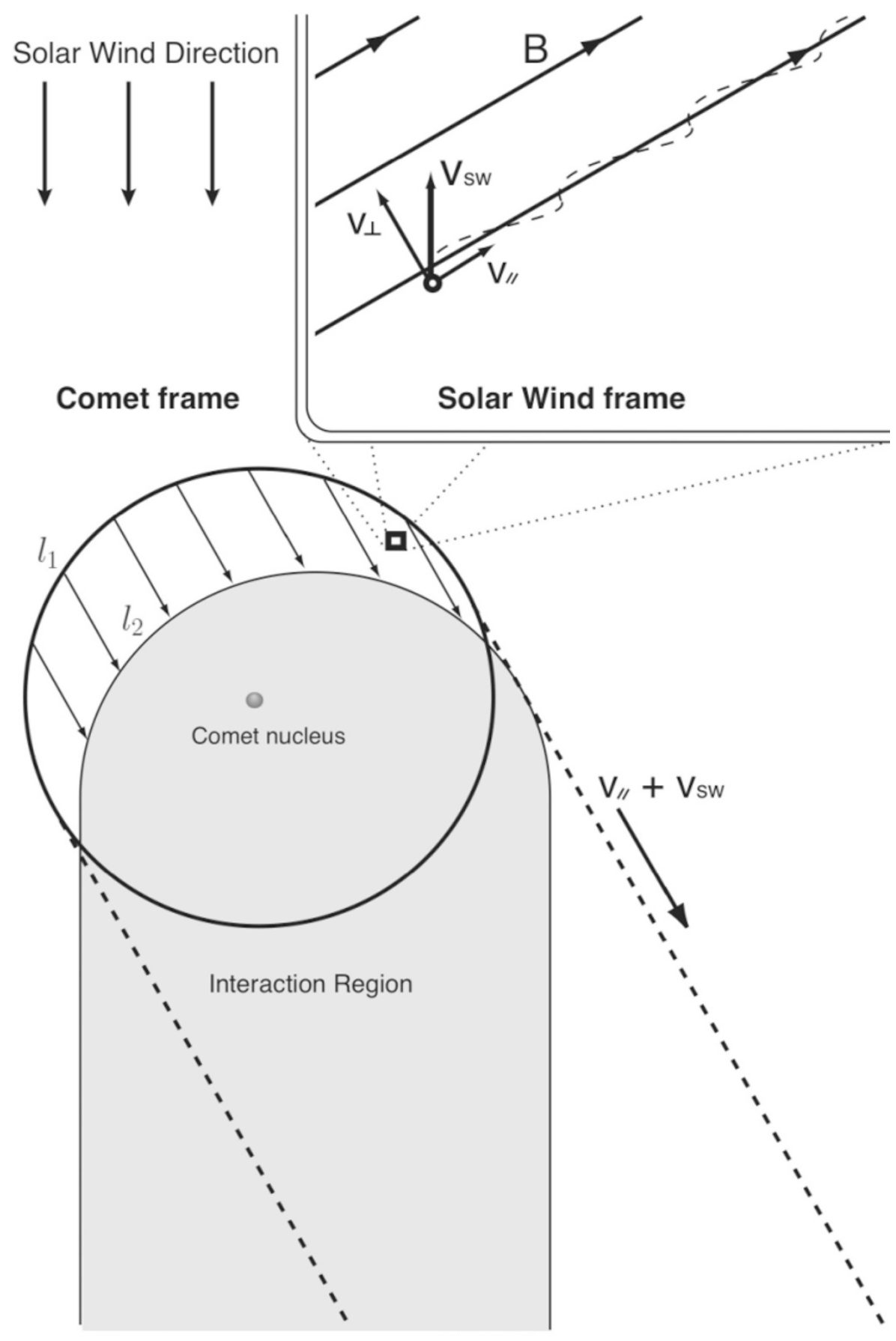

Fig. 1. Schematic diagram of asymmetric distribution of interaction region (after Oya, 1993). When the ionization of cometary particles takes place on the co-circular line $l_{1}$, the ions start to move in the direction of arrow which is controlled by the local magnetic field direction. The field aligned motion of cometary ions survive till merging into the solar wind. In this diagram, cometary ions stop their field aligned motion at the line $l_{2}$, and asymmetry is formed as described by shaded region.

experiments with a long time scale, which was difficult to be carried out for a decade because of the limitation of the computational resources and the technical problem due to suffering of numerical heating of ions through the long simulation running caused by small number of superparticles in the "particle in cell" (PIC) method. By using results of long time scale hybrid simulation in this paper, we discuss on the merging process of cometary ions into solar wind where spatial distribution of interaction region is deeply influenced by field aligned motion of picked up ions.

\section{Linear Theory}

We start from the evaluation of plasma instability in Comet-Coma region based on linear theory to find the critical spatial and time scale of the plasma instability. Four species of particles are taken into account, namely, protons and electrons of solar wind plasma, and o xygen ions and electrons of cometary origin. Our model uses a reference frame which is moving with solar wind plasma. In this frame, cometary origin particle has drift velocity with solar wind speed directed to anti-sunward direction, because solar wind velocity 
(a)

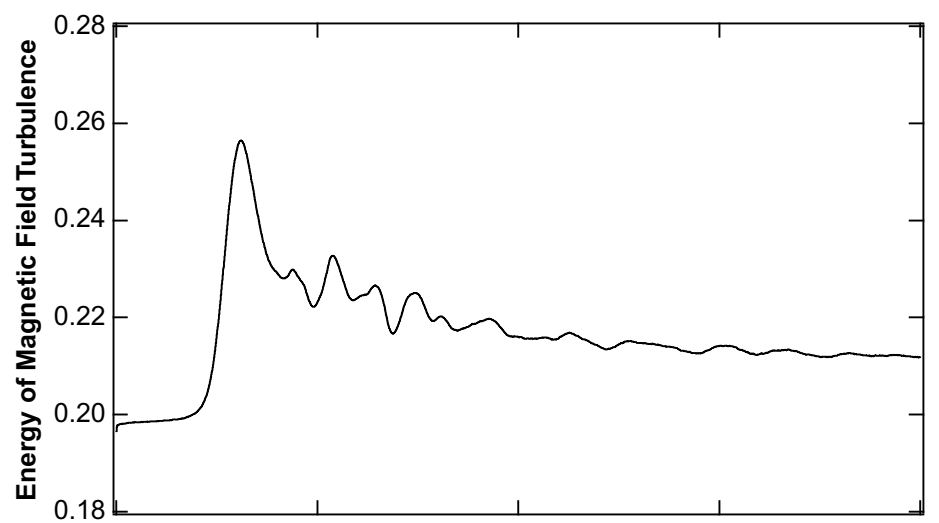

(b)

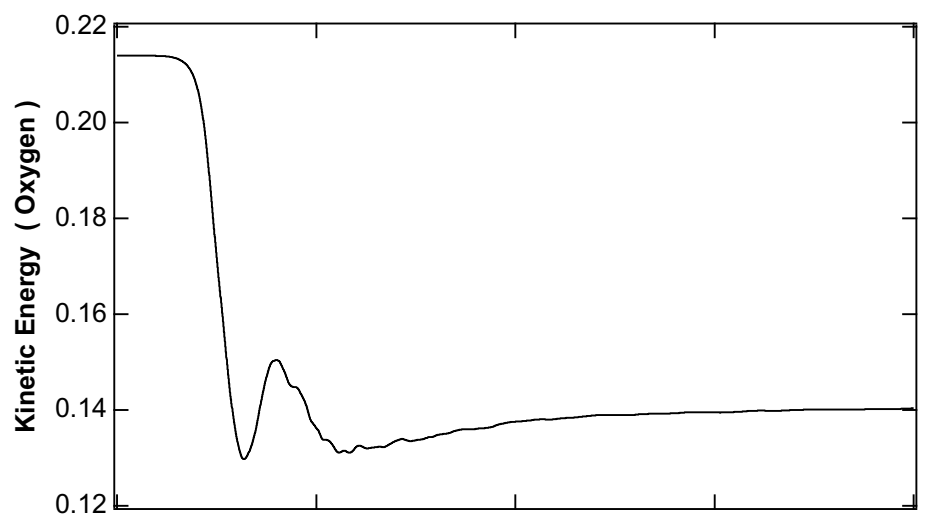

(c)

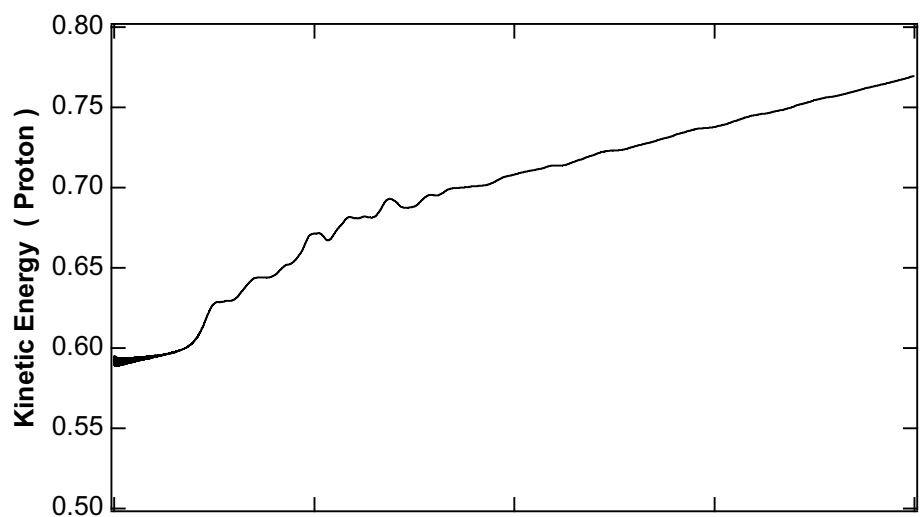

$(d)$

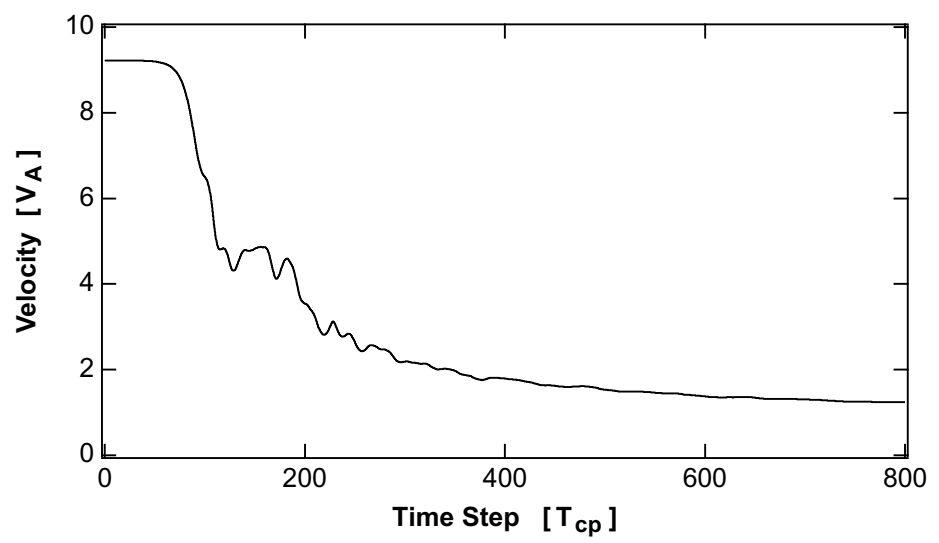

Fig. 2. Time history of (a) magnetic field energy, (b) kinetic energy of cometary ion, (c) kinetic energy of solar wind proton, (d) field aligned component of cometary ion velocity normalized by Alfvén velocity. Energies of magnetic field and particles are normalized by total energy at $t=0$. 


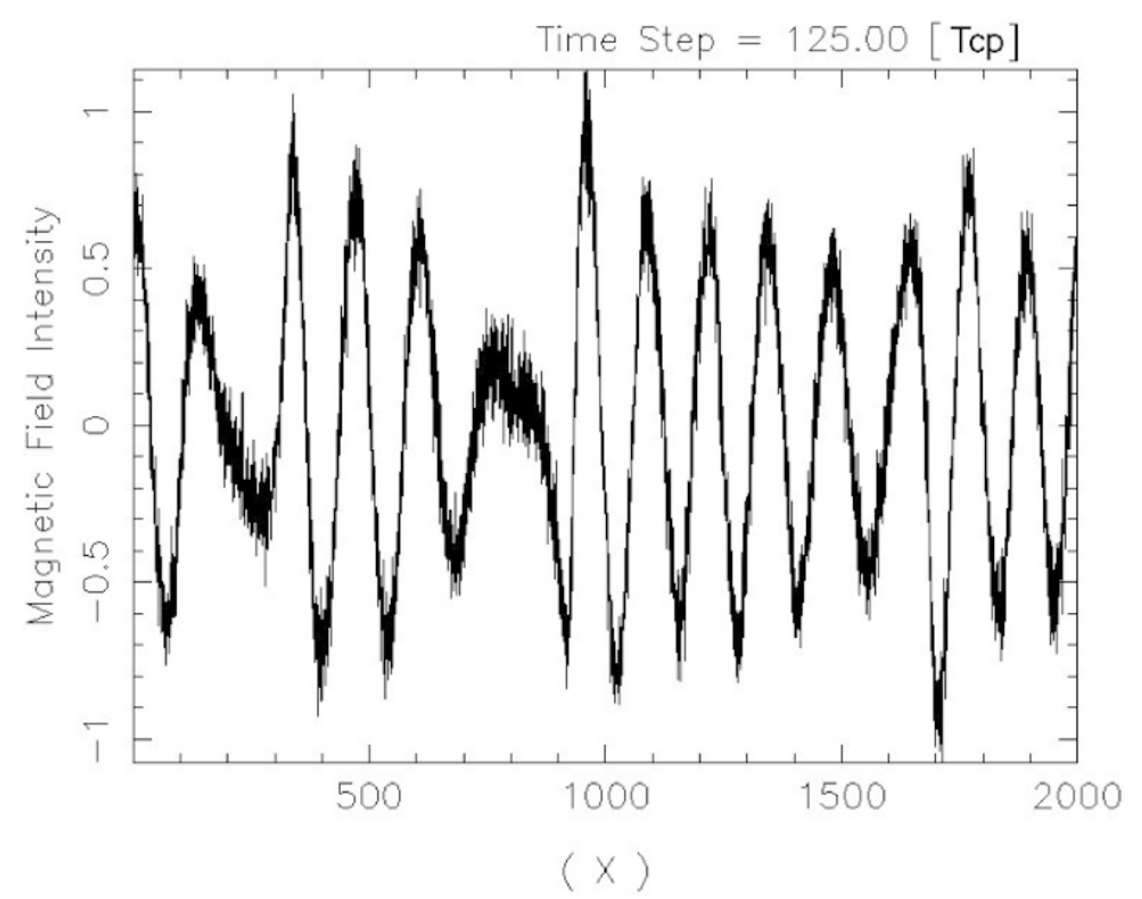

Fig. 3. Snap shot of Y component of magnetic field at $125 T_{c p}$. Large amplitude MHD wave is excited by ion beam instability with wave length 140 $V_{A} T_{c p}$.

is much greater than initial velocity of ionized cometary particles $(\sim 1 \mathrm{~km} / \mathrm{s})$. The beam drift velocity along the magnetic field $v_{b \|}$ is defined by $v_{b \|}=V_{S W} \cos \theta$, where $\theta$ is an angle between $B_{0}$ and $V_{S W}$. Although the modification of the instability in the case of the non-zero $\theta$ has been well investigated by several workers (e.g. Winske et al., 1985), in order to see detail of field aligned motion of cometary ions, here we examine a special case that solar wind flow is directed in parallel to the ambient magnetic field direction, $\theta=0^{\circ}$.

The plasma parameters used in this model are selected based on the results of in-situ observation at Comet-Coma region measured by Sakigake (Saito et al., 1986); namely, magnetic field intensity of $B_{0}=5 \mathrm{nT}$ and the solar wind velocity of $V_{S W}=450 \mathrm{~km} / \mathrm{s}$. The particle number densities are given as $n_{p}=n_{e}=5 \mathrm{~cm}^{-3}$, and $n_{O}=n_{E}=4 \times 10^{-3} \mathrm{~cm}^{-3}$, which are based on the model estimated by using observation results by Giotto (Wegmann et al., 1987). Temperature of particles are selected as $T_{p}=T_{e}=T_{E}=1 \times 10^{5} \mathrm{~K}$, $T_{O}=1.93 \times 10^{3} \mathrm{~K}$, where the subscripts $p, e$ denote the quantities associated with solar wind protons and electrons, and the subscripts $O, E$ denote the quantities of cometary origin oxygen ions and electrons, respectively. For the particle distribution functions of ions and electrons in the solar wind and cometary origin, a shifted bi-Maxwellian velocity distributions are assumed for each species.

Numerical calculations of the linear dispersion equation showed plasma instability of ion waves due to the cometary ion beams. The dispersion relation reveals that plasma waves are generated due to the ion beam instability, $\omega+k v_{b \|}=\Omega_{c o}$, where $\Omega_{c o}$ is gyrofrequency of oxygen ion, in the region $\omega: 2.34 \times 10^{-2} \Omega_{p}, k: 6.0 \times 10^{-3} R_{p}^{-1}$, where $R_{p}$ is the Larmor radius of solar wind proton, with the growth rate of $3.4 \times 10^{-3} \Omega_{p}^{-1}$. This solution is consistent with previ- ous studies that ion beam instability is the dominant factor of wave excitation when $\theta$ is nearly zero (e.g., Omidi and Winske, 1986), and this wave mode has Right-handed propagation mode. Wave length with the maximum growth rate is $138 v_{A} T_{c p}$ where $T_{c p}$ refers the gyro-period of proton; this results from the linear dispersion relation give an important parameter for the preparation of computer simulation scheme that the scale size of simulation system should be larger than this wave length.

\section{Hybrid Simulation}

To examine the nonlinear evolution of MHD waves, we utilize one dimensional hybrid simulation code, while ions are treated as particles while electrons are assumed to be a fluid with negligible mass. We adopt the periodic condition at the boundary of our model. The simulation frame is assumed to be moving with the solar wind velocity. Within the simulation frame, protons of solar wind origin and $\mathrm{O}^{+}$ions of cometary origin are taken into account, while electrons behave to establish the charge neutrality.

Basic equations are given as,

$$
\begin{gathered}
m_{i} \frac{d \boldsymbol{v}_{\boldsymbol{i}}}{d t}=q\left(\boldsymbol{E}+\boldsymbol{v}_{\boldsymbol{i}} \times \boldsymbol{B}\right)-\eta \boldsymbol{J} \\
\boldsymbol{v}_{\boldsymbol{i}}=\frac{d \boldsymbol{x}_{\boldsymbol{i}}}{d t} \\
\frac{\partial}{\partial t}\left(n_{e} m_{e} \boldsymbol{v}_{\boldsymbol{e}}\right)=-q n_{e}\left(\boldsymbol{E}+\boldsymbol{v}_{\boldsymbol{e}} \times \boldsymbol{B}\right)-\nabla\left(n_{e} k T_{e}\right)+n_{e} \eta \boldsymbol{J} \\
=0
\end{gathered}
$$


(a)

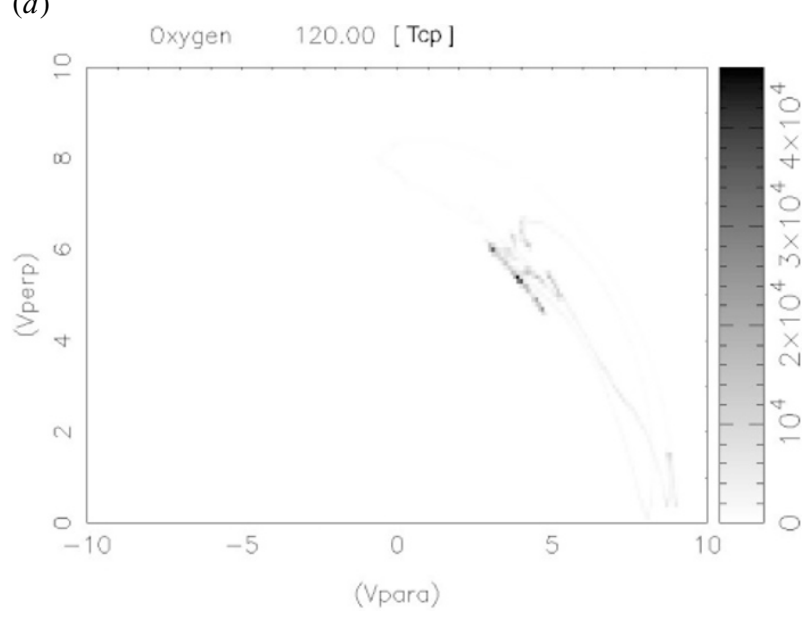

(c)

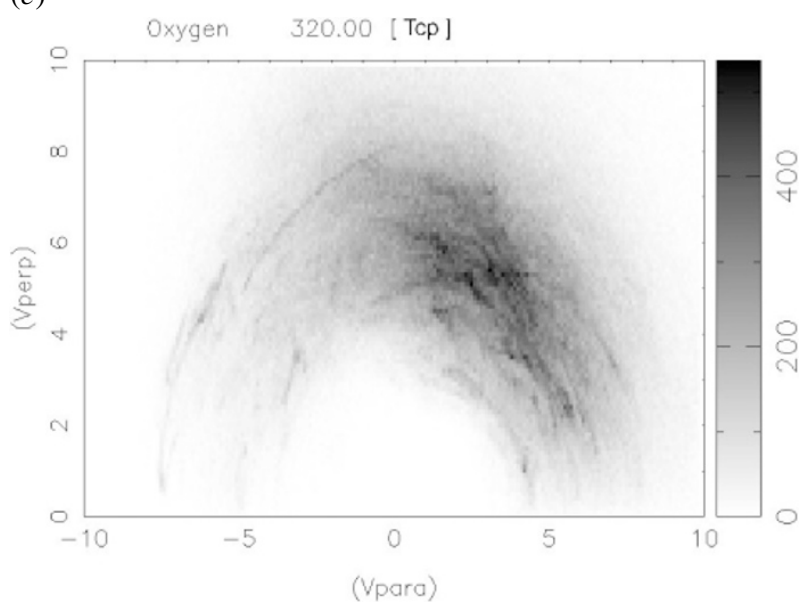

(b)

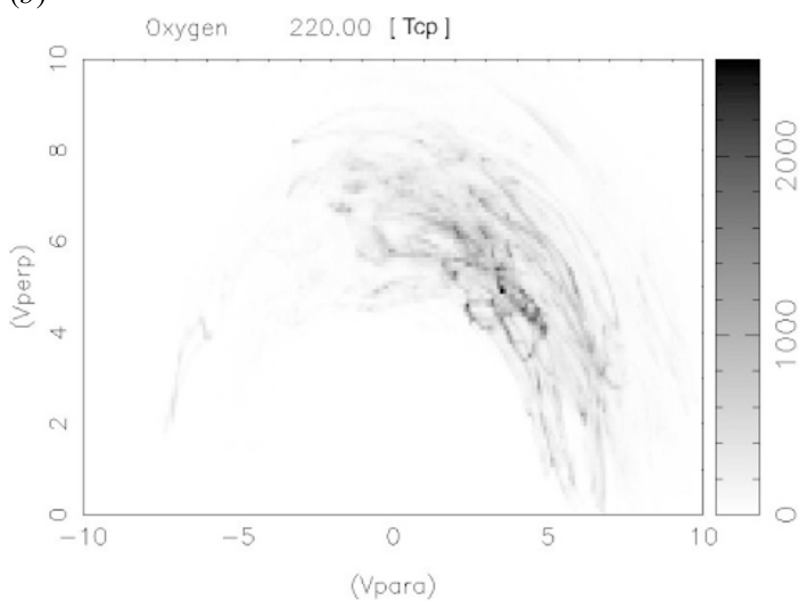

(d)

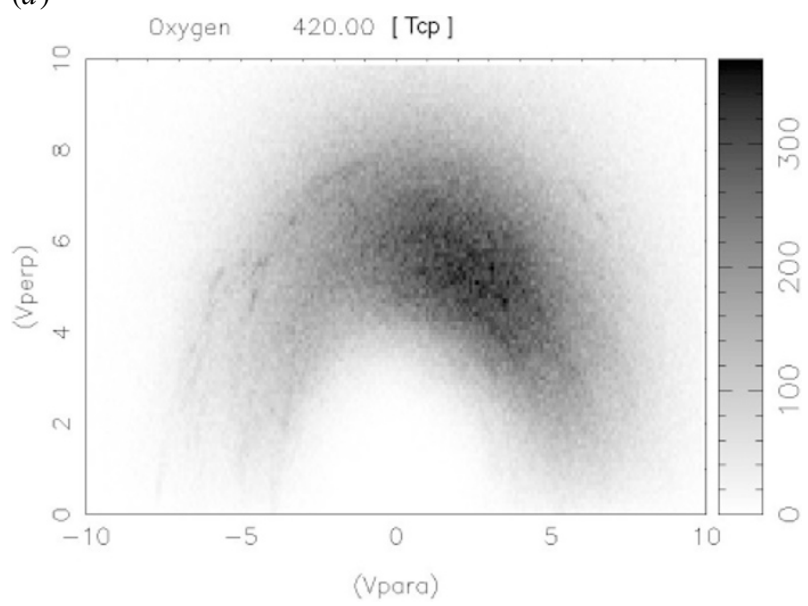

Fig. 4. Velocity distribution of cometary ions in $v_{\|}-v_{\perp}$ plane at (a) 120, (b) 220,(c) 320, (d) $420 T_{c p}$. After saturation of MHD wave amplitude, pitch angle of cometary ions is scattered and become isotropic.

$$
\begin{gathered}
\boldsymbol{J}=\frac{1}{\mu_{0}} \nabla \times \boldsymbol{B} \\
\nabla \cdot \boldsymbol{B}=0
\end{gathered}
$$

where the subscript $i$ denote the quantities associated with ions; solar wind proton and cometary oxygen ions, and the subscript $e$ denote the electrons, respectively. The constant $\eta$ in Eqs. (1) and (3) represent the viscosity of MHD plasma, however, it is assumed as $\eta=0$, because a mean free path is significantly larger than the scale size (about $10^{6} \mathrm{~km}$ ) of the present model.

To implement the simulation code, each physical values are normalized to be a dimensionless quantities, namely, time is normalized by proton gyro-period $T_{c p}$, and velocity and length are normalized by Alfvén speed $V_{A}$ and $V_{A} T_{c p}$, respectively. We choose grid length $\Delta \mathrm{x}$ as $0.4 V_{A} T_{c p}$ and time step $\Delta \mathrm{t}$ as $0.02 T_{c p}$, and system lengths $\mathrm{L}_{x}$ as $5000 \Delta \mathrm{x}$, which satisfy the time and spatial limits comparing with the results of the linear analysis.

Since the motions of both cometary ions and solar wind protons are treated by using PIC (Particle in Cell) method, we assumed the number of superparticles as 160 per grid that is relatively large number comparing with previous studies (e.g., Winske and Omidi, 1993) to avoid numerical thermalization of particles due to the long time scale simulation. We have confirmed that the numerical accuracy $(10 \%$ for the conservation of total energy) are conserved till time step of $800 T_{c p}$ by employing 160 superparticles.

Figure 2 shows the results of the hybrid simulation giving time progress of energies and the average field aligned velocity of cometary ions. The variation of the magnetic field energy is given in Fig. 2(a), while a significant growth of the magnetic field energy arises with the maximum intensity at the time step of $125 T_{c p}$. This is due to the excitation of large amplitude MHD wave. Figure 3 shows a snap shot of magnetic field fluctuation along the background magnetic field line at the time step of $125 T_{c p}$. Examining the plasma waves shown in Fig. 3, we can see plasma instability generating large amplitude MHD wave with the R-mode propagation character that is recognized as Fast-mode Alfvén wave. At time-step $125 T_{c p}$ in Fig. 2(d), excitation of large amplitude wave is associated with rapid decrease of the ion beam drifting parallel to the magnetic field.

Since amplitude of MHD wave reaches a saturation level at $125 T_{c p}$, the turbulence of magnetic field starts a grad- 


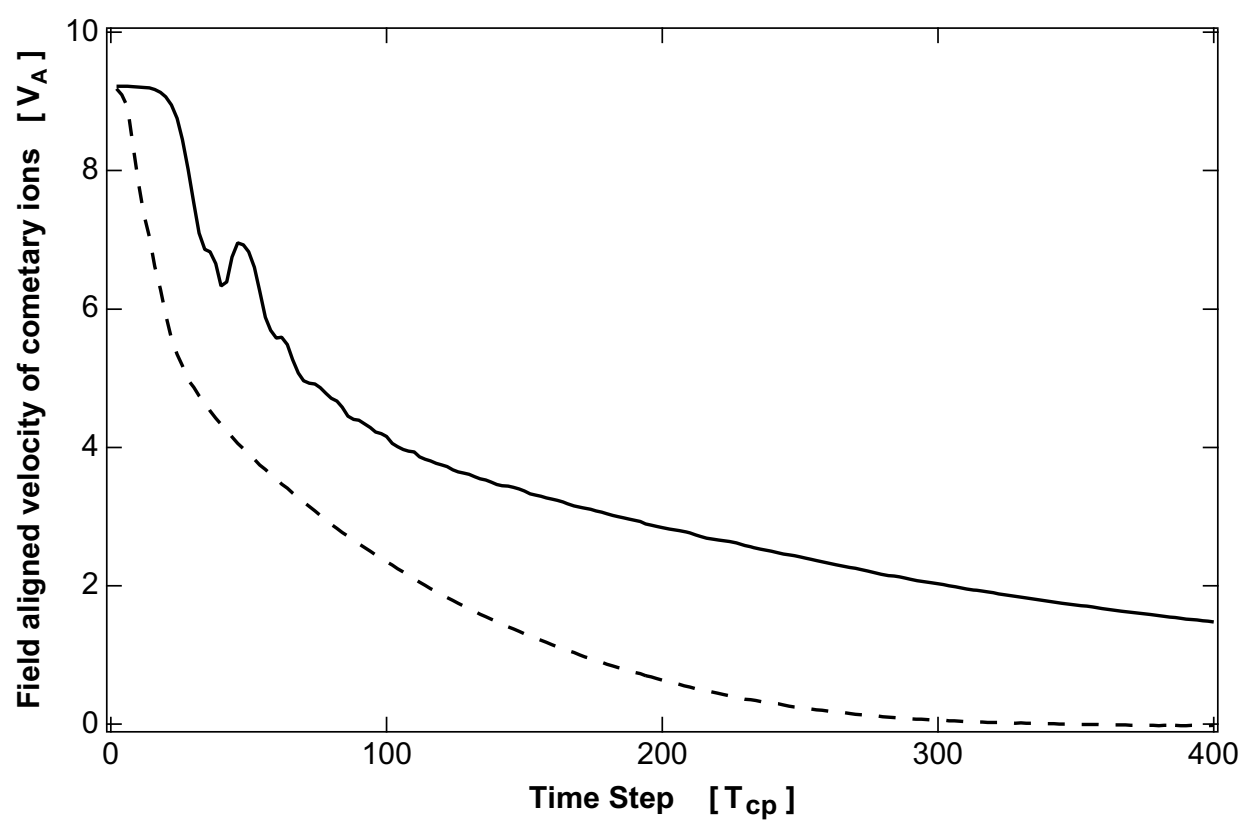

Fig. 5. Variation of field aligned velocity of pick-up ions in test simulations assuming helium (solid line) and proton ions (dashed line) for the cometary ions.

ual damping signature. Although kinetic energy of cometary ions converges to an appropriate value, field aligned component of cometary ion velocity still keeps decreasing signature continuously. Figure 4 shows velocity distribution of cometary ions in phase space at 120, 220, 320, $420 T_{c p}$. As it is shown in Fig. 4 that pitch angle of cometary ions is scattered and velocity distribution of cometary ions becomes isotropic that is similar to the shell distribution of cometary ions discovered in the Comet-Coma region (Mukai et al., 1986) which is explained by the pitch angle scattering of cometary ions (Wu et al., 1986; Gary et al., 1986). Based on the temporal change of the pitch angle distribution, it is concluded that a gradual decrease of the bulk velocity of oxygen ions is caused by the effect of pitch angle scattering.

\section{Summary and Discussion}

Simulation results show that field aligned drifting speed of cometary ion is decreased due to the energy transfer to the generating of MHD waves and initial pitch angle distribution is suffered by a significant scattering effect due to an effective collision process with MHD wave. Turbulent state of MHD waves also causes energization of background solar wind protons as it is shown in Fig. 2(c). Even after the saturation of MHD waves, pitch angle scattering of cometary ions is still sustained and the velocity distribution of ions is also kept changing forward to the isotropic character because turbulent state of MHD waves is still active due to the remainder of beam component of cometary ions. An effective collision process between cometary ions and MHD waves results pitch angle scattering of cometary ions as shown in Fig. 4, that is the major process of the decreasing of field aligned velocity of cometary ions as it has been shown in Fig. 2(d). Therefore, Figure 2 shows the acceleration process of cometary ion to the solar wind velocity due to the nonlinear wave particle interaction between the MHD waves and oxygen ions of comet origin.

At the time progress of $800 T_{c p}$, bulk velocity of cometary ions reaches nearly equal to Alfvén velocity. Although it is a little amount, there can be still seen a continuous decrease of the field aligned velocity component. To see the terminal stage of the ion pick-up process, we examine test simulations assuming helium and proton ions for the cometary ions. We tested by using ions because these simulation results show that light cometary ions excite MHD waves rapidly and also they are rapidly merged into solar wind clearly, as shown in Fig. 5, although intensity of MHD waves is lower than the case of oxygen. The results of these simulations showed that cometary oxygen ions are possibly merged into the solar wind finally. Since spatial scale of asymmetry of CometComa region is controlled by field aligned motion of pick up ions, we estimate spatial scale of field aligned motion of cometary ion from Fig. 2 (d) to $1.5 \times 10^{6} \mathrm{~km}$. Although this scale is rather small comparing with the scale of observed asymmetry, namely $5 \times 10^{6} \mathrm{~km}$, asymmetry of Comet-Coma region by Sakigake was observed in a condition where the angle between solar wind flow and magnetic field was nearly $45^{\circ}$. This condition suggests that intensity of MHD wave was lower than the case of present study because of initial speed of field aligned motion of cometary ion was lower than that of solar wind. Low wave activity causes a tendency that field aligned motion of cometary ions survive for a long time, so it is inferred that spatial extent of the turbulent region becomes larger than that of the present case of $\theta=$ $0^{\circ}$. Therefore, it should be said that the spatial scale of the interaction region which is examined in the present paper is the minimum case. Although a two dimensional simulation is required to study the more realistic case of non-zero $\theta$, the physical basis of the merging process of cometary ions would be same as studied in the present paper.

As a summary, we conclude that the braking process of 
the field aligned motion of picked up ions are understood by the wave particle interaction through the excitation of the MHD wave and pitch angle scattering caused by the turbulent magnetic field, and cometary ions are finally merged into the solar wind plasma flow. The result of the present paper supports the scenario that observed asymmetrical distribution of the interaction region of the comet is formed by the motion of picked up ions toward the field aligned direction until their merging into the solar wind, as it was proposed by Oya (1993).

Acknowledgments. The base of this research was built by master thesis of Mr. Takashi Inaba, Tohoku University, 1996. Numerical computation in the present work were performed at the Information Synergy Center of Tohoku University and at the Information Science Center, National Institute of Polar Research, Japan. Effort of T. Ono is supported by the 21 th Century COE program by the Ministry of Education, Culture, Sports, Science and Technology, Japan. The authors thank D. Winske and another referee for valuable comments and discussions.

\section{References}

Gary, S. P., C. D. Madland, D. Schriver, and D. Winske, Computer simulations of electromagnetic cool ion beam instabilities, J. Geophys. Res., 91, 4188-4200, 1986

Karimabadi, H., D. Krauss-Varban, N. Omidi, S. A. Fuselier, and M. Neugebauer, Low-frequency instabilities and the resulting velocity distributions of pickup ions at comet Halley, J. Geophys. Res., 99, 21541-21556, 1994

Kojima, H., H. Matsumoto, Y. Omura, and B. T. Tsurutani, Nonlinear evolution of high frequency R-mode waves excited by water group ions near comets: computer experiments, Geophys. Res. Lett., 16, 9-12, 1989.

Krauklis, I. and A. D. Johnstone, The turbulent state of cometary ion pickup generated fluctuations near comet P/Halley, J. Geophys. Res., 103,
12005-12014, 1998

McKenna-Lawlor, S., E. Kirsch, D. O'Sullivan, A. Thompson, and K. P. Wenzel, Energetic ions in the environment of comet Halley, Nature, 321, 347-349, 1986.

Mukai, T., W. Miyake, T. Terasawa, M. Kitayama, and K. Hirao, Plasma observation by Suisei of solar-wind interaction with comet Halley, Nature, 321, 299-303, 1986.

Omidi, N. and D. Winske, A kinetic study of solar wind mass loading and cometary bow shocks, Geophys. Res. Lett., 13, 397-400, 1986.

Omidi, N. and D. Winske, Simulation of the solar wind interaction with the outer regions of the coma, J. Geophys. Res., 92, 13409-13426, 1987.

Oya, H., Interaction of plasma of Halley's comet with the solar wind, in Primitive Solar Nebula and Origin of Planets, edited by H. Oya, pp. 579614, 1993.

Saito, T., K. Yumoto, K. Hirao, T. Nakagawa, and K. Saito, Interaction between comet Halley and the interplanetary magnetic field observed by Sakigake, Nature, 321, 303-307, 1986.

Terasawa, T., M. Hoshino, J.-I. Sakai, and T. Hada, Decay instability of finite-amplitude circularly polarized Alfvén waves: a numerical simulation of stimulated Brillouin scattering, J. Geophys. Res., 91, 4171-4187, 1986.

Wegmann, R., H. U. Schmidt, W. F. Huebner, and D. C. Boice, Cometary MHD and Chemistry, Astron. Astrophys., 187, 339-350, 1987.

Winske, D. and N. Omidi, Hybrid codes: methods and application, in Computer Space Plasma Physics: Simulation Techniques and Software, edited by H. Matsumoto and Y. Omura, 103 pp, Terra Scientific Publishing Company, Tokyo, 1993.

Winske, D., C. S. Wu, Y. Y. Li, Z. Z. Mou, and S. Y. Guo, Coupling of newborn ions to the solar wind by electromagnetic instabilities and their interaction with the bow shock, J. Geophys. Res., 90, 2713-2726, 1985.

Wu, C. S., D. Winske, and J. D. Gaffey, Jr., Rapid pickup of cometary ions due to strong magnetic turbulence, Geophys. Res. Lett., 13, 865-868, 1986 .

Y. Katoh (e-mail: yuto@kurasc.kyoto-u.ac.jp), H. Oya, M. Iizima, and T. Ono 